\title{
Aspergillus flavus as a surprise space occupying lesion in an immunocompetent pediatric patient
}

\author{
Mir Ibrahim Sajid ${ }^{1 *}$ (D), Noor Malik², Samira S. Balouch ${ }^{3}$ and Ehsan Bari ${ }^{2}$
}

\begin{abstract}
Background: Fungal infections of the CNS are almost always a clinical surprise. Aspergillus species although ubiquitous are more frequently observed in immuno-compromised individuals upon inhalation of conidia. Most of the fungal infections which happen in humans are opportunistic, mostly due to immunocompromised host.

Case description: We present the case of a 14-year-old boy who presented to the Emergency Department of Aga Khan University Hospital, Karachi, with complaints of right-sided weakness of the body. This was a rapid-onset condition which was associated with gait disturbances and multiple episodes of vomiting. MRI head showed encapsulated Space Occupying Lesion with puss in the left frontal lobe with surrounding edema. The patient was planned for a craniotomy to remove an intracerebral abscess. Histopathology report revealed the presence of chronic granulomatous inflammation with necrosis and numerous septate hyphae. A fungus culture was run which confirmed the presence of heavy colonies of Aspergillus flavus.

Conclusion: Fungal infections of the central nervous system are almost always a clinical surprise, have a subtle presentation and mistaken often as meningitis, brain abscess, or tumor. Any suspected lesion once removed should be sent for biopsy to rule out the presence of any fungal infection.
\end{abstract}

Keywords: Immunocompetent host, Frontal brain mass, Fungal infection, Aspergillus flavus

\section{Background}

Fungi are usually occurring organisms in the environment with only a few being pathogenic. Most of the fungal infections which happen in humans are opportunistic, mostly due to immunocompromised host. However, some fungal infections can happen, even in normal hosts. Fungal infections of the central nervous system are almost always a clinical surprise, have a subtle presentation, and mistaken often as meningitis, brain abscess, or tumor [1].

Though Aspergillus species vary in color, size, and growth rate, nevertheless microscopic characteristics are fairly uniform across all species. For example, all have hyphae that are septate and hyaline. Aspergillus gets its

\footnotetext{
*Correspondence: ibisajid@gmail.com

'The Aga Khan University, Stadium Road, Karachi 74880, Pakistan

Full list of author information is available at the end of the article
}

name from the shape: vesicle in the shape of a circle, with filamentous extensions growing out from it [2]. Fungi are categorized and classified based on morphology and molecular genetics. Aspergillus species although ubiquitous are more frequently observed in immuno-compromised individuals upon inhalation of conidia. The most usual complications are lung and $\mathrm{cu}-$ taneous infections [3].

Fungal infections can be a cause of neurosurgery or can occur following a neurosurgical procedure. Postsurgical aspergillosis is an uncommon complication that carries a high mortality rate in affected patients. Given how rare the outcome is, the diagnosis is challenging, since it requires highly sensitive methods to isolate Aspergillus from surgical sites, especially in brain tissues [4]. 


\section{Case presentation}

We present the case of a 14-year-old boy, a known case of asthma, who presented to the Emergency Department (ED) of Aga Khan University Hospital, Karachi, with complaints of right-sided weakness of the body. This was a rapid-onset condition which was associated with gait disturbances. The parents told that the child had multiple episodes of vomiting since the past 2 days and constant headache since a week. While the patient was in the emergency room, he suffered an attack of seizure which was initially conservatively managed.

On examination, he was person-oriented, however, lack of time and place orientation. Magnetic resonance imaging (MRI) head showed encapsulated Space Occupying Lesion (SOL) with an abscess in the left frontal lobe with surrounding edema (as shown in arrows in Fig. 1b). With the suspicion of a tumor which most likely looked like high-grade glioma, the patient was referred to neurosurgery (Fig. 1a-c).

The patient was planned for a craniotomy to remove an intracerebral abscess. Peri-operative findings revealed a non-vascular, hard, encapsulated lesion in the left frontal lobe which was medially extending to the falx cerebri. With no residual abscess, the entire lesion was resected. The brain sample was sent for a detailed histopathology report. Post-operatively the patient was shifted to special care unit and was started on IV amphotericin, IV paracetamol, and IV hydrocortisone to prevent the recurrence of the fungal infection and resolution of inflammation and edema.

The gross description of the biopsy revealed single light brown color nodular tissue that measures approximately $3 \times 2 \mathrm{~cm}$. The cut surface was light brown and homogenous. Microscopic examination was carried out using Periodic acid-Schiff (PAS) special stain which revealed the presence of numerous septate fungal hyphae.
With the diagnosis of chronic granulomatous inflammation with necrosis and numerous septate hyphae, according to microbiology report, serum galactomannan, serum 1, 3-beta-D-glucan was negative. Gram stain revealed few pus cells and rare septate hyphae and fungus culture confirmed the presence of heavy colonies of Aspergillus flavus (Fig. 2). An acid-fast bacilli (AFB) stain was run negative for any such growth.

The patient remained stable and was discharged on the fourth day of surgery on oral voriconazole, dexamethasone, levetiracetam, and paracetamol. After a week of follow-up of discharge, the patient had completely recovered from his neurological symptoms and resumed his normal daily activities.

\section{Discussion}

Aspergillus flavus infection in the brain parenchyma can present as sudden onset headache and loss of consciousness, with or without any other symptoms of systemic infection such as convulsions, abnormal movements, eye abnormalities, vision impairment, drowsiness, weakness, fever, vomiting, or skin rashes. However, a patient can complain of facial pain, swelling, weakness, and impaired or compromised cranial nerve. Sometimes, a palpable mass can be felt during the physical examination which could prompt a physician to investigate the presenting symptoms with the lesion; however, since the mass of our patient was deep in the brain matter, it could not be palpated [5-7].

Imaging modalities such as CT scans and an MRI are used to localize the lesion and measure the extent of the damage. Newer modalities such as endoscopic endonasal approaches are also being used to visualize the lesions. The suspected mass can be visualized in any part of the brain, e.g., occipital lobe, frontal lobe, and the parasellar region with extension to other parts of the brain.

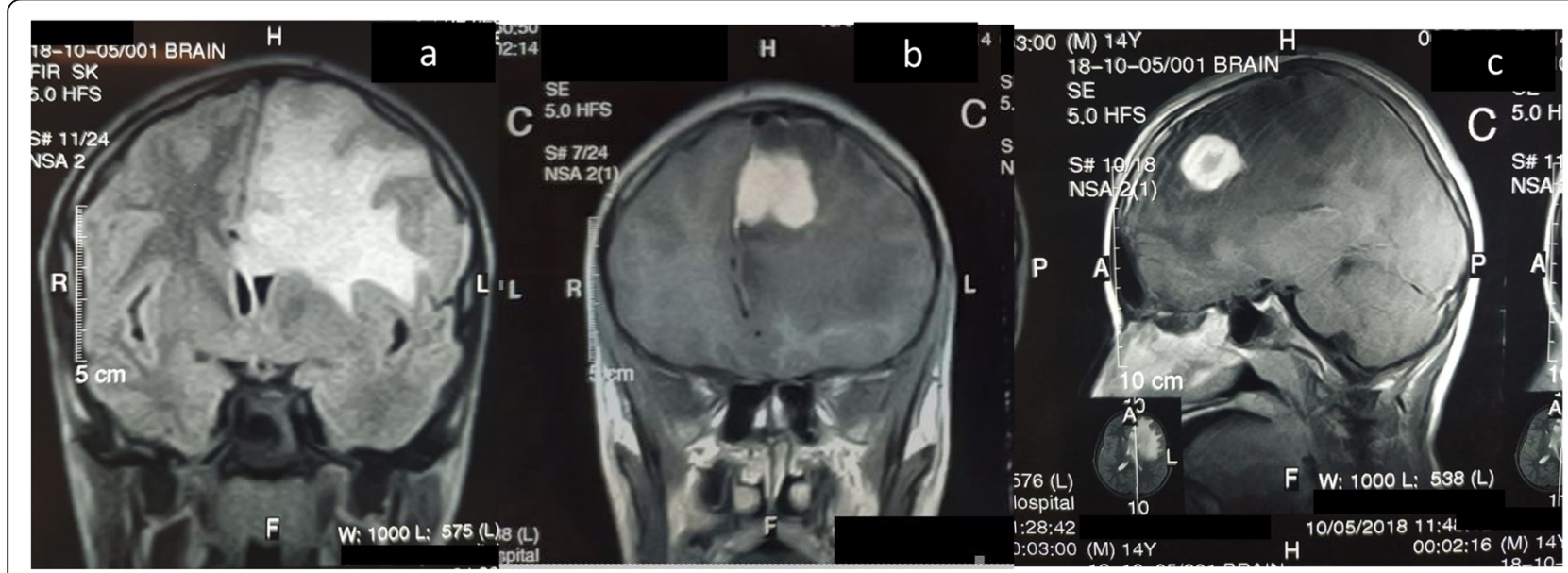

Fig. 1 MRI showing Space Occupying Lesion with surrounding abscess and edema 


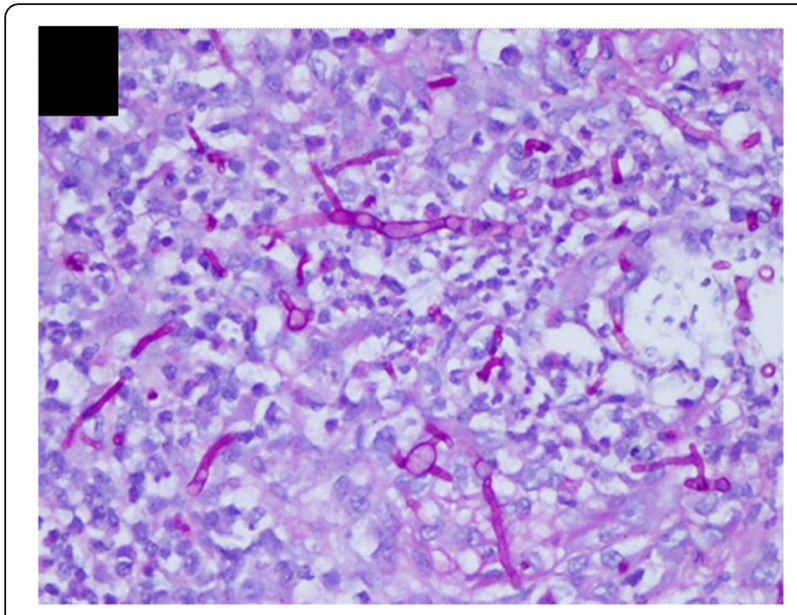

Fig. 2 Periodic acid-Schiff (PAS) special stain which revealed the presence of numerous septate fungal hyphae

However, infection of the sinus is also seen with the primary mass both being in the sinus and protruding to the brain or vice versa $[6,8]$. In our patient, the mass was however localized to the frontal lobe and did not extend into any sinus.

Usually, the lesion in the brain has a heterogeneous enhancement and is surrounded with peri-focal edema as in our case. Imaging can also show involvement of the soft tissue with midline shift to the opposite side of the mass. However, the tumor can also cause destruction of the facial bones, the cribriform plate, and invade the facial sinuses. Involvement of the pachymeningeal layers can result as a result of the abovementioned conditions, which however were spared in our patient $[5,6]$.

Histopathology examination is the key to the etiological diagnosis of the mass, including any fungal infection. Microscopic examination can reveal many hyphae engulfed by multinucleated giant histiocytes and marked eosinophilia. The hyphae in the case of Aspergillus being hyaline septate hyphae with dichotomous, regular acute angle branching and vesical arising from conidiophores [7] (Fig. 2).

Fungal infections are mostly a manifestation in immunocompromised individuals who have co-morbidities such as neutropenia, uncontrolled hypertension, or diabetes mellitus. However, a fungal infection in an immunocompetent person is a rare finding and should be thoroughly investigated as in our case [8].

\section{Conclusion}

Fungal infections of the central nervous system are almost always a clinical surprise and have a subtle presentation and mistaken often as meningitis, brain abscess, or tumor. Any suspected lesion once removed should be sent for biopsy to rule out the presence of any fungal infection.

\section{Acknowledgements}

N/A

\section{Authors' contributions}

MIS did manuscript writing, NM procured patient data, SSB proofread the manuscript, and EB supervised the project. The authors read and approved the final manuscript.

\section{Funding}

No funding was obtained for this project.

\section{Availability of data and materials}

The patient's data was pulled through the Health Information Management Systems of Aga Khan University Hospital after permission from the parents.

\section{Declarations}

Ethics approval and consent to participate

Ethical approval has been sought from the IRB: Ethics and Review

Committee (ERC) of The Aga Khan University, Karachi, Pakistan.

\section{Consent for publication}

Parental consent has been obtained and will be made available on the request of the editor.

\section{Competing interests}

The authors declare that they have no competing interests.

\section{Author details}

${ }^{1}$ The Aga Khan University, Stadium Road, Karachi 74880, Pakistan. ${ }^{2}$ Section of Neurosurgery, Department of Surgery, The Aga Khan University Hospital, Karachi, Pakistan. ${ }^{3}$ Section of Oral and Maxillofacial Surgery, Department of Surgery, King Edward Medical University, Lahore, Pakistan.

Received: 31 October 2019 Accepted: 15 April 2021

Published online: 03 May 2021

References

1. Nadkarni T, Goel A. Aspergilloma of the brain: an overview. Postgrad Med J. 2005;51(5):37.

2. Aspergillus - microbewiki [Internet]. Microbewiki.kenyon.edu. 2018 [cited 8 December 2018]. Available from: https://microbewiki.kenyon.edu/index. php/Aspergillus.

3. Vadlapudi V, Borah N, Yellusani KR, Gade S, Reddy P, Rajamanikyam M, et al. Aspergillus Secondary Metabolite Database, a resource to understand the secondary metabolome of Aspergillus genus. Sci Rep. 2017;7(1):7325. https://doi.org/10.1038/s41598-017-07436-w.

4. Gonzales Zamora J, Henry Z, Gultekin S. Central nervous system aspergillosis: an unexpected complication following neurosurgery. Diseases. 2018;6(2):46. https://doi.org/10.3390/diseases6020046.

5. Al-Maskari N, Hussain I, Jumaa S, Al-Shail E. Aspergillus flavus -induced brain abscess in an immunocompetent child: case report. Sultan Qaboos Univ Med J. 2016;16(2):e246-9. https://doi.org/10.18295/squmj.2016.16.02.019.

6. Jariwal R, Heidari A, Sandhu A, Patel J, Shoaepour K, Natarajan P, et al. Granulomatous invasive Aspergillus flavus infection involving the nasal sinuses and brain. J Investig Med High Impact Case Rep. 2018;6: 232470961877047. https://doi.org/10.1177/2324709618770473.

7. Punia P, Goel N, Singh I, Chaudhary U. Occupational CNS aspergillosis in an immunocompetent individual a diagnostic challange. Arch Environ Occup Health. 2017;73(6):1-4.

8. Saffarian A, Derakhshan N, Taghipour M, Eghbal K, Roshanfarzad M, Dehghanian A. Sphenoid Aspergilloma with headache and acute vision loss. World Neurosurg. 2018;115:159-61. https://doi.org/10.1016/j.wneu.2018.04. 054.

\section{Publisher's Note}

Springer Nature remains neutral with regard to jurisdictional claims in published maps and institutional affiliations. 